\title{
The enigmatic origins of CHON: unraveling the isotopic clues in primitive matter
}

\section{KEVIN D MCKEEGAN}

University of California - Los Angeles

Presenting Author: mckeegan@epss.ucla.edu

Carbon, hydrogen, oxygen, and nitrogen comprise four of the five most abundant elements in the galaxy and, along with sulfur and phosphorous, form the major group of life-essential elements. Given their abundance and long-recognized astrophysical and astrobiological importance, it is somewhat ironic that our understanding of the origins and distributions of source reservoirs of C-H-O-N in the solar system, and even on the Earth, has remained a challenging and elusive goal. For four decades, Francois Robert has been at the vanguard of the study of isotopic tracers to reveal the enigmatic origins of $\mathrm{CHON}$ in the solar system. Increasingly sophisticated analyses have shown that the isotopic threads are complexly intertwined in solar system materials, even in the organic matter of so-called primitive carbonaceous meteorites which Robert and Epstein first investigated 40 years ago [1].

This presentation will discuss progress in understanding the origins and evolutionary histories of $\mathrm{CHON}$ in the rocky planets and their precursor planetesimals. The pursuit of primitive matter has extended from acid-residues of asteroidal samples to analyses of cometary components, including cosmic dust collected at Earth and particles returned to terrestrial laboratories by the Stardust mission as well as gases analyzed in situ in the coma of comet $67 \mathrm{P} /$ Churyumov-Gerasimenko. Isotopic evidence pointing to inheritance of $\mathrm{CHON}$ components from interstellar reservoirs must be understood within the context of mixing processes in the solar nebula, taking into account knowledge of the average solar nebula composition as represented in the Sun through the analyses of captured solar wind. A key to further progress in understanding inherited compositions and solar system processes affecting isotopic memories would be precise and accurate analyses of the light element isotopic compositions of cometary (and planetary) ices, whether through in situ spacecraft or via sample return to terrestrial laboratories. So far, no robust analyses of isotopic compositions of ices from the outer solar system exist; rectifying this situation should be among the highest priorities for mission development in the next decade.

[1] Robert \& Epstein (1982). The concentration and isotopic composition of hydrogen, carbon and nitrogen in carbonaceous meteorites. Geochimica et Cosmochimica Acta, 46, 81-95. 